\title{
Optimizing Energy and Modulation Selection In Multi-Resolution Modulation For Wireless Video Broadcast/Multicast
}

\author{
James She ${ }^{1}$, Pin-Han Ho ${ }^{1}$ and Basem Shihada ${ }^{2}$ \\ 1 University of Waterloo, Waterloo, ON, N2L 3G1, Canada, \\ \{james,pinhan\}@bbcr.uwaterloo.ca, \\ WWW home page: http://bbcr.uwaterloo.ca/ james/ \\ 2 King Abdullah University of Science and Technology, Thuwal 23955-6900, \\ Kingdom of Saudia Arabia
}

\begin{abstract}
Emerging technologies in Broadband Wireless Access (BWA) networks and video coding have enabled high-quality wireless video broadcast/multicast services in metropolitan areas. Joint source-channel coded wireless transmission, especially using hierarchical/superposition coded modulation at the channel, is recognized as an effective and scalable approach to increase the system scalability while tackling the multiuser channel diversity problem. The power allocation and modulation selection problem, however, is subject to a high computational complexity due to the nonlinear formulation and huge solution space. This paper introduces a dynamic programming framework with conditioned parsing, which significantly reduces the search space. The optimized result is further verified with experiments using real video content. The proposed approach effectively serves as a generalized and practical optimization framework that can gauge and optimize a scalable wireless video broadcast/multicast based on multi-resolution modulation in any BWA network.
\end{abstract}

Key words: wireless video broadcast/multicast, energy allocation, scalable video coding, superposition coding, optimization

\section{Introduction}

Unprecedented advancements in 3/4G Broadband Wireless Access (BWA) networks based on Long Term Evolution (LTE), IEEE 802.11n and IEEE 802.16 (WiMAX) standards as well as scalable video coding technologies, such as H.264/MPEG4 Advanced Video Coding (AVC), have made it possible in provisioning large-scale and high-quality wireless video broadcast/multicast applications, such as mobile/wireless Internet Protocol Television (IPTV), wireless digital signage, e-poster, etc. Adopting the use of wireless broadcast/multicast radio signals for these broadcasting/multicasting applications achieves the best scalable usage of transmission capacity at the base stations (BSs). Instead of the number of receivers, the system scalability is only determined by the number of video channels simultaneously provisioned along with their bandwidth 
requirements. This facilitates the possible largest-scale and highest-quality wireless video broadcast/multicast, in which multiple receivers simultaneously receive bandwidth-intensive data of the same video stream from the same broadcast/multicast radio signal. One of the legacy problems in the aforementioned approach is the transmission rate selection under multi-user channel diversity. A broadcast/multicast signal using monotonic modulation rate could under-utilize the channel capacity of some receivers with good channel conditions, while being not decodable by some receivers with bad channel conditions. A straightforward yet dummy solution could be adopting the most conservative transmission rate for satisfying as many receivers as possible. This results with the expense of a much reduced number and quality of video channels that can be jointly provisioned, which certainly leads to a poor economic scale. To tackle such multi-user channel diversity problem, efficient and robust cross-layer architectures for scalable wireless video broadcast/multicast are recently emerged [1-11], which will be described in the following section. Among these skillfully engineered cross-layer architectures, multi-resolution modulated broadcast/multicast radio signals are commonly generated through hierarchical/superposition coded modulation at the channel. Each resolution of such modulated signal embeds a successively refinable quality layer of the original video data that is encoded by a scalable video coding. Allocating optimal energies and modulation schemes for different quality layers of video data are the common and critical operational decisions in these cross-layer approaches. However, it is completely lack of a generalized optimization framework that gives the global optimum in high computational efficiency. This motivates the contributions of our work to introduce a generic optimization framework for energy allocation and modulation selection for this rapidly growing area in wireless video broadcast/multicast. For the simplicity, only the word of "multicast" is referred in most of the following discussions, since all the concepts, technical details and our proposed solution are basically equivalent or interchangeable in both scenarios of wireless video broadcast and multicast. The paper is organized as follows. Previous works and some backgrounds are described in Section II. Section III details a generic system model and the proposed optimization framework. The section is divided into four subsections to thoroughly detail the performance metric, optimization variables, objective function and proposed optimization framework. Section IV provides the results of optimizations performed using the developed framework followed by Section V, where a conclusive discussion is summarized there.

\section{Previous Works and Background}

\subsection{Previous Works}

Novel cross-layer architectures of scalable wireless video multicast are actively introduced in recent years to address the multi-user channel diversity problem through multi-resolution modulated multicast signals coupled with successive refinable source coding. One of our earliest works [1] presented the results from 
the extensive simulations using real video trace files of High-definition Television (HDTV) content. It proved the effectiveness of this cross-layer approach not just in preserving the use of multicast for higher system scalability. A much better video quality is offered to all receivers all the time, while assuring the base video quality regardless of their average channel conditions. However, formulations and optimizations of energy allocation and modulation selection are not further discussed, which are critical to these kinds of cross-layer architectures. Initial effort on the energy allocation problem was made by Sesia et al. in [2], the optimization problem was formulated by discretizing the continuous fading states, and an algorithm was devised when the source coding layers are assumed with the same rate. This algorithm, however, does not directly yield the optimal energy allocation when the fading states are discrete and pre-specified, nor does it give a closed-form solution for the continuous case. Etemadi et al. also considered this problem in [3], and provided an iterative algorithm by separating the optimization problem into two sub-problems. However, explicit modulation schemes are not considered in their optimization algorithm. In two interesting recent works [4], [5], $\mathrm{Ng}$ et al. provided a recursive algorithm with the worsttime complexity of $O\left(2^{M}\right)$ to compute optimal energy allocations across quality layers under finite fading states, where $M$ is the number of fading states. However, it minimizes the distortion in terms of mean-squared errors, which is based on a system model assuming a Gaussian source. Since a video content is never with a Gaussian distribution, such approach can only serve as an analytical purpose but not practically work for a real-world deployment and operation. One of our previous works [6] have developed a generic system model without any assumption of source distribution and incorporated practical modulation schemes for the optimization. The total received bitstreams of each wireless multicast signal is considered as the performance metric, which will be maximized by optimal energy and modulation scheme for achieving the highest video quality. A 3 -fold iterative search is introduced to determine optimal allocations of energy and modulation scheme throughout the entire solution space. Some other recent works $[7,8,9]$, including those from the industry such as Qualcomm [10, 11], also considered some additional parameters in their proposed cross-layer designs along with the multi-resolution modulations for scalable wireless video multicast. Up to our knowledge, however, there is no any previous attempt in this area to obtain the global optimum through the dynamic programming with a much reduced solution space at a linear complexity of $O(n)$. This paper is the first attempt to introduce such efficient optimization framework that generically applicable to many, if not all, cross-layer architectures for scalable wireless video multicast based on multi-resolution modulation.

\subsection{Background - Superposition Coded Multicast}

For the purpose of simplicity, the proposed optimization framework is based on a generic cross-layer designed wireless video multicast architecture - Superposition Coded Multicast (SCM) [1] shown in Fig. 1, in which a sample scenario of wireless video multicast with two quality layers is assumed. In fact, the optimization 
framework is designed with a great flexibility to accommodate any arbitrary number of quality layers and even other integrated parameters. It is applicable to other similar architectures of the interests under any total energy transmit limit and collection of modulation schemes.

There are 7 major procedures in SCM to generate and decode a wireless video multicast signal, which has multi-resolution modulated data embedding multiple video quality layers:

(1) A video frame or group of frames (GoF) is encoded by a scalable video encoder into scalable bitstreams with multiple quality layers (e.g., base and enhancement layers).

(2) Video bitstreams of each quality layer is queued into individual buffer for the multi-resolution modulation.

(3) Hierarchical/superposition coded modulation [12, 13, 14] is adopted in this procedure to generate multi-resolution modulated signal. Both of them are well-studied modulation technique for broadcast in the community of information theory. Some background idea is described in the next sub-section. According to the selected modulation, $M_{i}$, for layer $i$, corresponding number of bits in buffer $i$ is modulated individually with an allocated energy $E_{i}$. The modulated signal is then superimposed with another signal modulated with energy $E_{j}$ from buffer $j$, where $i \neq j$. Note that the order of modulation scheme selected for layer $i$ must not be higher than that for layer $j$ due to the intrinsic mechanism of multi-resolution modulation, where $i<j$.

(4) SCM signals containing bitstreams of base and enhancement layers are multicasted over the wireless channel.

(5) A receiver obtains a SCM multicast signal, and decodes the signal according to its channel condition using the technique of signal-interference cancelation that will be described in the following.

(6) Depending on the receiver's channel condition, a receiver generally can decode the base layer bitstream for a basic video quality. If the receiver's channel condition is good enough, more bitstreams of the enhancement layer can be decoded from the same received SCM signal for a higher video quality.

(7) Decoded bitstreams from each layer are reassembled to reconstruct the video at the receiver's video output, as illustrated in Fig.1.

In summary, the procedure (3) involves the operational parameters, i.e., energy, $E_{i}$, and modulation schemes, $M_{i}$, to be optimized in each multi-resolution modulated multicast signal for maximizing the video quality.

\subsection{Background - Hierarchical/Superposition Coded Modulation}

The intrinsic goal of hierarchical/superposition coded (SPC) modulation is to facilitate a transmitter to send two or multiple independent receiver's information within a single wireless radio signal by superimposing two or multiple modulated signals at the channel level. The superposition of two modulated signals is analogous to the vector addition in a signal constellation diagram. 


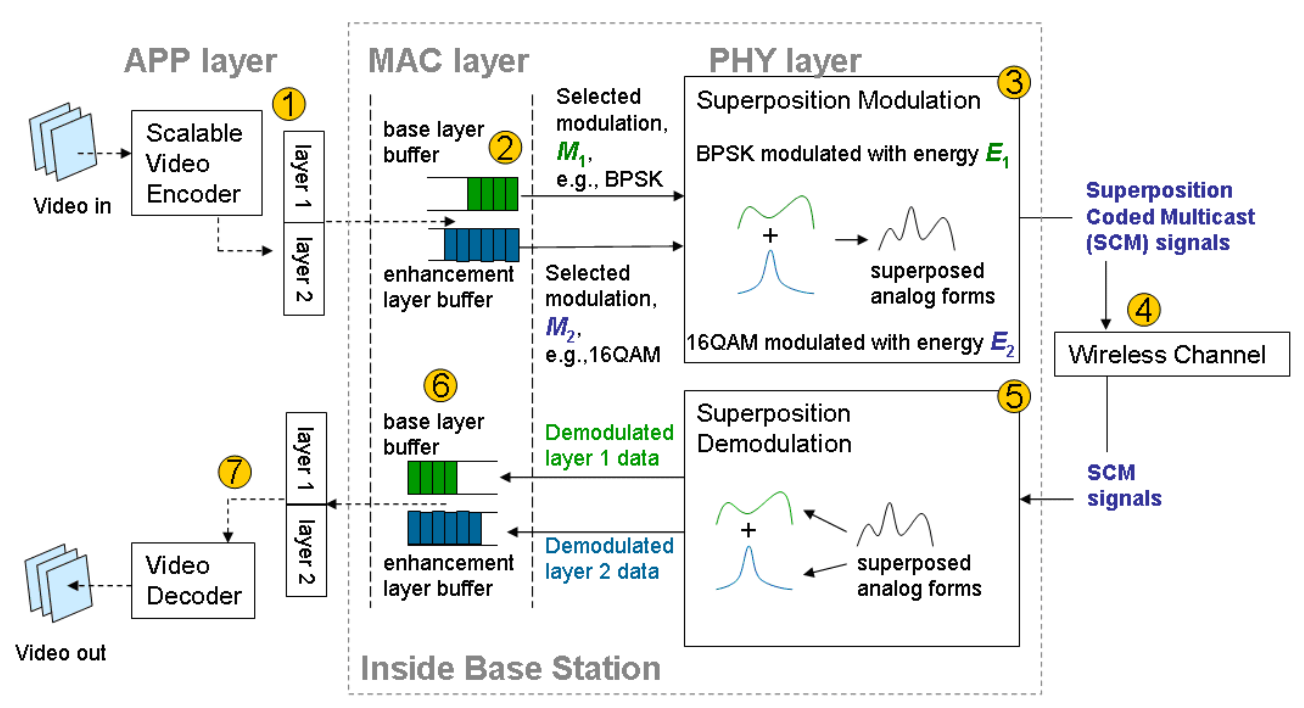

Fig. 1. An overview of SCM for wireless video multicast.

As shown in Fig. 2, $x_{1}$ with the information for receiver 1 is modulated using QPSK for a higher transmission rate yet weaker resistance to channel noises, and $x_{2}$ with the information for receiver 2 is modulated by BPSK (a lower order of modulation scheme) for stronger resistance to channel noises but slower transmission rate. The superimposed signal, $x$, is a vector sum of the two modulated signals governed by $x=x_{1}+x_{2}$. In Fig. 2(c), vector $x$ represents the superimposed signal, consisting of symbol ' 0 ' from Fig. 2(b) and symbol '01' from Fig. 2(a). The signal $x$ is then launched as a single wireless broadcast/multicast transmission signal and received by two receivers with diverse channel conditions within the same coverage.

The received signal is expressed as $y_{i}=x+z_{i}$, where $z_{i}$ is the noise perceived by receiver $i$. The conventional technique to decode the SPC modulated multicast signals is known as Signal-Interference Cancelation (SIC), which is used at receiver $i$ to identify the signal components meant for the noise and other receivers. Receiver $i$ obtains its own information by subtracting those noncorresponding signal components from its received signal $y_{i}$. For example, for receiver 1 to decode its data from $y_{1}$, it must first use SIC to determine the data meant for receiver $2, x_{2}$, and then subtract $x_{2}$ from the received signal $y_{1}$. The result of the subtraction using SIC is $x_{1}$, which is usually distorted by the noise experienced at receiver 1 , i.e., $z_{1}$. Note that $x_{2}$ is completely decoded by receiver 1 during the process of obtaining $x_{1}$ using SIC. The information of $x_{2}$ in fact could be utilized by receiver 1 , if it refers to the basic quality of video as discussed in this work. 


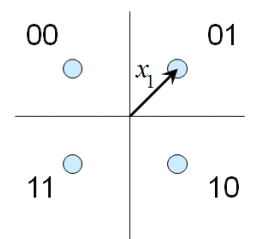

(a)

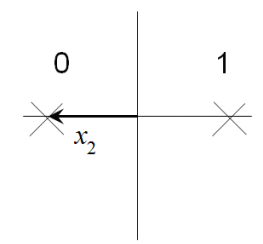

(b)

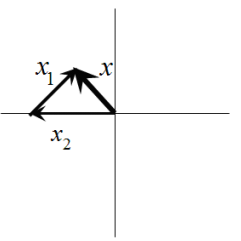

(c)

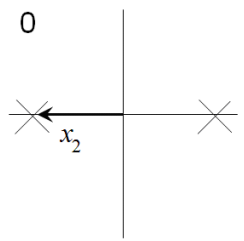

(d)

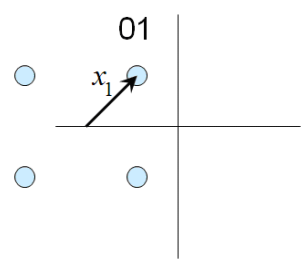

(e)

Fig. 2. (a-c) SPC modulation, and (d-e) SPC demodulation.

\section{The System Model And Optimization Framework}

The system model is common in a number of ways to many related previous works, which requires optimized energy allocation and modulation selection under certain transmit energy limit.

\subsection{Performance Metric - Total Received Bitstreams}

Video quality is always challenging, if not impossible, to be measured quantitatively, due to the subjective importance of content perception in each video frame. According to the rate-distortion theory, a monotonically decreasing trend of the distortion, $D\left(b_{i}\right)$, with an increasing number of bits received is observed as shown in Fig. 3 . The bit boundary, $b_{i}$, indicates the minimum amount of bitstreams required for the video quality corresponding to layer $i$.

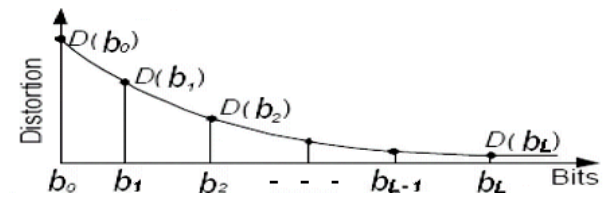

Fig. 3. Distortion vs. bitstream boundaries in a frame or a $\mathrm{GoF}$

It is proved in [6], the amount of total received bitstreams, $T$, of a frame or a GoF decodable by a receiver can serve as an objective and measurable approximation of the perceived video quality. Hence, it is used as the performance metric of the objective function in the optimization. With a sample scenario of 
two quality layers (i.e., $L=2$ ) in this paper, the total received bitstreams, $T$, is accounted by the received bitstreams of base and enhancement layers, $T_{1}$ and $T_{2}$, which can be formulated as:

$$
T=\sum_{i=1}^{L} T_{i}
$$

\subsection{B. Variables - Energy Allocation Ratio and Modulation scheme}

The performance metric, $T$, in a scalable wireless video multicast architecture based on SCM is subject to the channel conditions, in terms of signal-to-noise ratios (SNRs), for receiving associated SCM multicast signals. In fact, the SNR, $\gamma_{i}$, of the channel condition in receiving bitstream of layer $i$ by a receiver, is determined by the following optimization variables.

Allocation ratio, $\beta$ : The energy $E_{i}$ allocated for modulating the bitstream of layer $i$ in each SCM signal is defined with the following condition:

$$
\begin{array}{r}
E_{1}(\beta)=(1-\beta) E \\
E_{2}(\beta)=\beta E
\end{array}
$$

where $E$ is the total energy transmit limit, and $\beta$ is the energy allocation ratio for $E_{1}$ and $E_{2}$. Note that a smaller $\beta$ value allocates less energy to the enhancement layer. The SNR, $\gamma_{i}$, of the channel condition in receiving bitstream of layer $i$ by a receiver relates to $E_{i}$ as follows:

$$
\gamma_{i}(\beta)=\frac{c d^{-\alpha}\left|h^{2}\right| 10 \log _{10} \frac{E_{i}(\beta)}{n_{0}}}{1+c d^{-\alpha}\left|h^{2}\right| \sum_{j=i+1}^{L} 10 \log _{10} \frac{E_{j}(\beta)}{n_{0}}}
$$

where the distance $d$ of the receiver from the BS, $\alpha$ is a path loss exponent ( $\alpha=4$ for urban area, or 3 for rural area), $c$ is some constant, $h$ is a random number from a Rayleigh fading distribution, and the averaged background noise, $n_{0}$, towards each transmit symbol. The variables $E_{1}$ and $E_{2}$ are indeed jointly optimized through the allocation ratio, $\beta$.

Modulation scheme, $M_{i}$ : the modulation/demodulation scheme selected for modulating/demodulating bitstreams in layer $i$ by a receiver has the following conditions:

$$
M_{i}\left(\gamma_{i}\right)=\left\{\begin{array}{r}
2(B P S K), \quad \text { if } 8 d B \leq \gamma_{i}<14 d B \\
4(Q P S K), \quad \text { if } 14 d B \leq \gamma_{i}<20 d B \\
16(16 Q A M), \text { if } 20 d B \leq \gamma_{i}<26 d B \\
64(64 Q A M), \text { if } 26 d b \leq \gamma_{i}
\end{array}\right\}
$$

By selecting a particular modulation $M_{i}$ for layer $i$ that fulfills the required SNR by a receiver channel, the symbol error, $e_{i}$, of decoding layer $i$ in each SCM signal can be derived as below: 


$$
e_{i}\left(M_{i}, \gamma_{i}\right)=\left\{\begin{array}{rrr}
Q\left(\sqrt{2 \gamma_{i}}\right), & \text { if } & M_{i}=1 \\
1-\left(1-2(1-1 / \sqrt{M}) e^{-3 \gamma_{i} / 2(M-1)}\right)^{2}, & \text { if } & M_{i}=2,4,6
\end{array}\right\}
$$

\subsection{The Objective Function}

Given the number of transmissions, $N$, required to multicast a GoF of a 2-layer video as well as other system parameters as shown in Table I for a wireless video multicast network, the amount of bitstreams, $T_{i}$, of layer $i$ successfully received by a receiver is derived as:

$$
T_{i}\left(M_{i}, \gamma_{i}\right)=N \log _{2}\left(M_{i}\right) \prod_{j=1}^{i}\left(1-e_{i}\left(M_{i}, \gamma_{i}\right)\right)^{N}
$$

Note that the existence of a product term in Eq. (6) is due to the decoding dependency of a higher layer on the lower layer required by the video decoder.

A multicast signal is shared by all receivers simultaneously within the coverage, and all receivers should able to decode some bitstreams from each multicast signal for the basic video quality. An objective function can be optimized for maximizing Eq. (1) (i.e., the video quality) subject to a multicast receiver with the worst-channel condition over the duration of a GoF or a reasonable long timewindow. The optimization problem can be formulated through the variables, $\beta$ and $M_{i}$ below:

$$
\max _{\beta, M_{i}} T=\sum_{i=1}^{L} T_{i}
$$

with the constraints of Eqs. (2)-(5) and $L=2$.

Table 1. Settings of a wireless video multicast network

\begin{tabular}{cr}
\hline Parameter & Setting \\
\hline$n_{0}$ & 1 \\
$E$ & 10000 (i.e., \\
$N$ & $40 \mathrm{~dB}$ ) \\
$c$ & 50 \\
& 1 \\
\hline
\end{tabular}

\subsection{The Proposed Optimization Framework}

Due to the discreteness and nonlinearity of Eqs. (4) and (5), it is intractable to solve the formulation optimally in Eq. (7) using simple optimization techniques. Note that a base layer have 4 discrete modulation schemes $\left(M_{i}=1,2,4,6\right)$ with a continuous value of energy allocation ratio $(1-\beta)$, and the enhancement layer 
also have 4 possible modulation schemes to pair with another continuous value of its allocation ratio, $\beta$. A dynamic programming (DP) can assure to obtain the global optimum under each allocation ratio, $\beta$, but involve a cost of parsing through a huge search space repeatedly. A 3-fold heuristic search methodology is therefore designed with the following steps, which reduces the search space significantly by conditioned parsings:

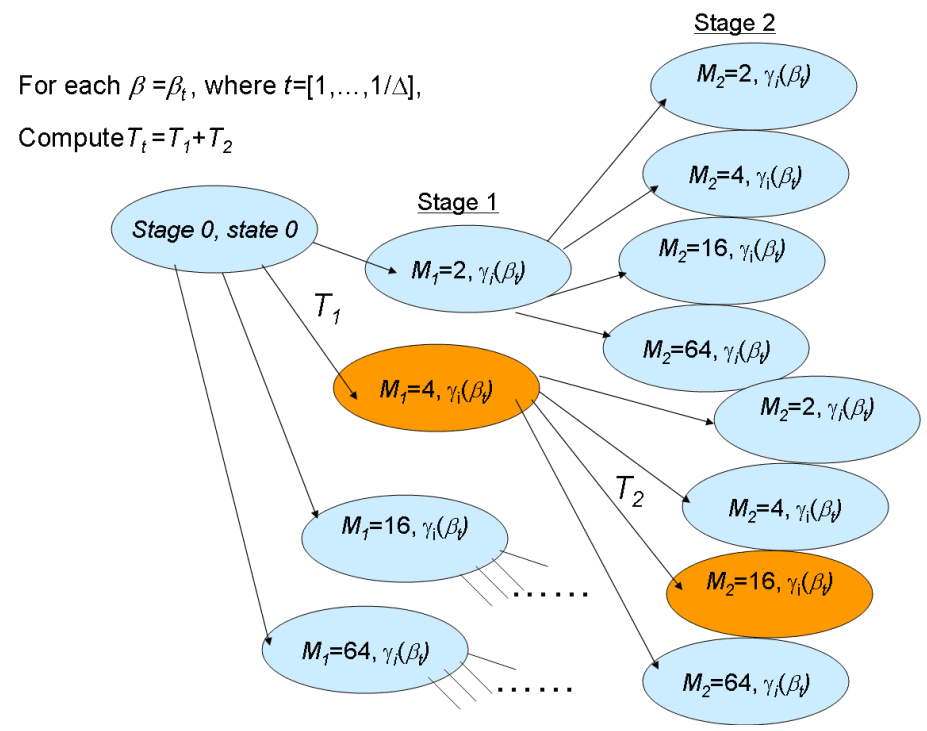

Fig. 4. Conditioned parsing of the DP graph for each value of $\beta_{t}$

(1) Discretizes the range of $\beta$ values evenly through a fixed interval, $\delta$, and take all discrete values to form a limited set with each of them denoted as $\beta_{t}$, where $t=[1, \ldots, 1 / \delta]$. Under many practical total energy transmit limits, for example, $\delta=0.001$ is already small enough to provide a BS generating a SPC multicast signal with the common SNR range from 0dB to $30 \mathrm{~dB}$. A smaller interval, $\delta$, is only required when a smaller granularity of energy value is necessary by a BS system for energy allocations over more quality layers or other operational factors.

(2) For each discrete $\beta_{t}$ value, a DP is conducted with the following definitions and conditioned parsings.

(a) Each stage of DP is referred as a quality layer (i.e., only stage 1 and stage 2 needed in our sample scenario.)

(b) Each state of stage $i$ corresponds to a possible modulation scheme, $M_{i}$, to be selected under a SNR, $\gamma_{i}$, subject to a discrete energy ratio, $\beta_{t}$.

(c) The benefit of each state is the received bitstream, $T_{i}\left(M_{i}, \gamma_{i}\left(\beta_{t}\right)\right)$ under selected $M_{i}$ and $\beta_{t}$. 
(d) Recall the rate-distortion theory discussed in Section II, it is desirable to receive as many video bitstream as possible reliably when the channel fulfills the SNR requirement of a modulation scheme. The parsing in each DP graph only considers the state with the highest value of $M_{i}$ that the channel SNR, $\gamma_{i}\left(\beta_{t}\right)$ supports with the constraint $M_{i} \leq M_{j}$ (where $i<j$ ) required by SCM. Hence, only two states will be parsed along each graph as shown in Fig. 4.

(e) $T_{t}=T_{1}+T_{2}$ is tracked and compared, where $t=[1, \ldots, 1 / \delta]$.

(3) The optimal energy allocation is given by the parse giving the maximum benefit $\left\{T_{t}\right\}$, where $t=[1, \ldots, 1 / \delta]$.

In summary, the search space for solving Eq. (7) is dramatically reduced to the complexity of $O(n)$, where $n=1 / \delta$ defined by a required granularity of energy values. The computation for optimal energy allocation and modulation selection is linearly efficient for a practical implementation.

\section{Experimental Results}

The optimal energy and modulation scheme allocations are optimized through the proposed optimization framework to maximize the video quality in a wireless video multicast network based on the settings in Table I. A general trend is observed that the perceivable SNR in a layer is proportional to the allocated energy on that layer. Hence, SNR is naturally expected to increase as the energy allocated in that layer increases as shown in Fig. 5(a). As less energy is allocated to the base layer when $\beta$ increases, a receiver only sustains a lower SNR that supports a lower order modulation with slower but more reliable transmission rate, and vice versa. Fig. 5(b) exhibits such dynamics of supportable modulation in each layer with respect to $\beta$.

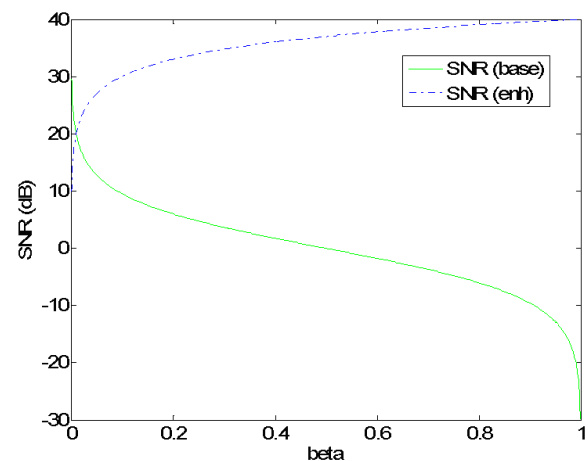

(a)

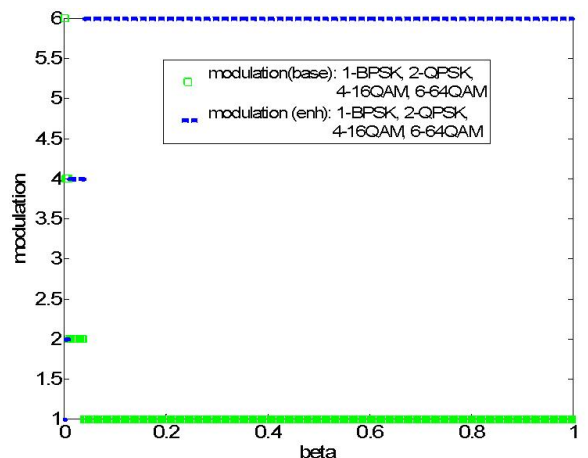

(b)

Fig. 5. Illustration of (a) SNR, and (b) optimal modulation selection, for each layer with respected to $\beta$ 
Fig. 6 indicates that the optimal energy allocation ratio is located at $\beta_{t}^{*}=0.134$ (i.e., $E_{2}: E_{1}=0.134: 0.866$ ) with optimal modulation selections as BPSK and 64QAM for the base and enhancement layers. These optimized settings give the largest amount of receivable bitstreams, $T$, from both layers (i.e., the highest video quality) over $N$ consecutive transmissions in a scalable wireless video multicast network using SCM signals.

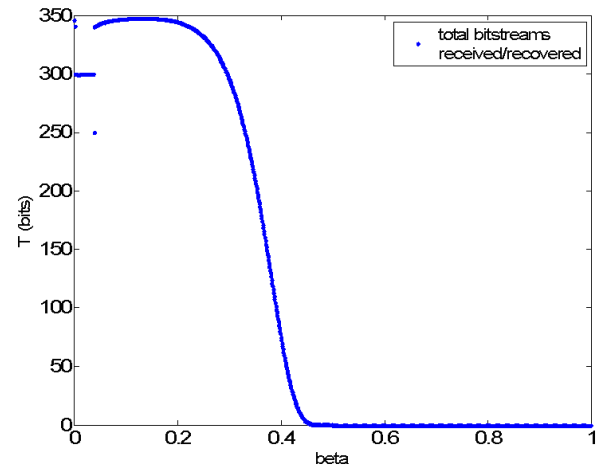

Fig. 6. Total received bitstream, $T$, vs. allocation ratio, $\beta$

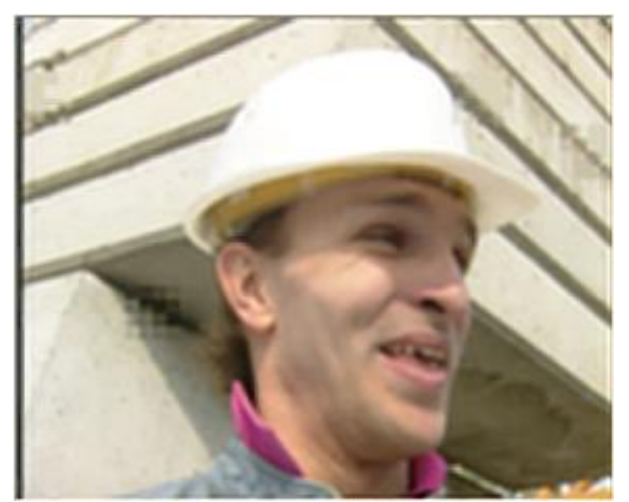

(a) $\beta_{t}^{*}=0.134$ when $\delta=0.001$

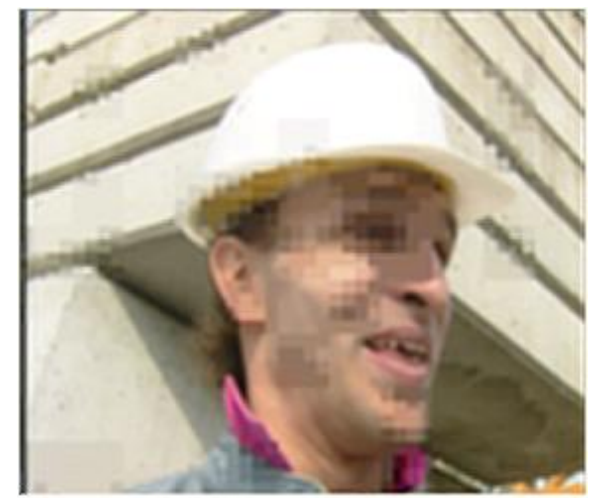

(b) $\beta_{t}^{*}=0.25$ when $\delta=0.25$

Fig. 7. Video qualities.

There is no any similar optimization framework previously available at this moment for comparing the search complexity and its impact towards the video quality. However, experimental simulations are realistically conducted in this work with some standard video sequence (in CIF format) using the optimized energy and modulation scheme allocations under various granularity of $\delta$ values. 
The purpose is to evaluate the computational cost and impact of $\delta$ values on the visual perception due to the resulting discretized $\beta_{t}^{*}$ values. As demonstrated in Fig. 7(a), a much better video quality is achieved with $\beta_{t}^{*}=0.134$ when $\delta=0.001$. Under the same optimized modulation selections as in Fig.7(a), the same video with a less perceptual quality is resulted in Fig. 7 (b) with $\beta_{t}^{*}=0.25$ when $\delta=0.25$ that exhibits a much faster computation. For a PC running with a $3.2 \mathrm{GHz}$ Pentium CPU and 1G RAM, it only takes about 0.0003 seconds (timed in the Matlab platform) to search the optimized configuration for $\delta=0.25$, but it is about 0.0623 seconds (i.e., $\approx 200$ times more) for $\delta=0.001$. This provides us an insight for the real-world deployment about the potential tradeoff of video quality with respect to the search complexity (i.e., the computational cost), which is determined by the $\delta$ value.

\section{Discussions And Conclusions}

It is demonstrated that the proposed DP optimization framework can find the global optimum configuration of energy and modulation scheme in a linear complexity of $O(n)$, which results with the highest amount of received bitstreams (i.e., the highest achievable video quality) even the optimization problem is discrete and nonlinear. Although it involves a certain number of graph parsings in multiple DP parsing processes, the optimization framework can efficiently determine the optimized variables by removing unnecessary search spaces through the conditioned parsing in each stage of every DP graph. The results from the optimization framework are further verified with the experiments on some real video sequence using optimized variables, which provided an useful and controllable insight on the tradeoff between the highest achievable video quality and computational cost. The current framework does not consider the sub-carriers assignment in each multicast/broadcast transmission, which are considerably useful and critical to 4G/BWA technologies. Up to our knowledge, this paper is the first attempt to develop such generalized framework for optimizing energy allocation and modulation selection that generically applicable to emerging cross-layer architectures for scalable wireless video broadcast/multicast in any coming BWA network.

\section{References}

1. J. She, F. Hou, P.-H. Ho and L.-L. Xie, "IPTV over WiMAX: Key Success Factors, Challenges, and Solutions", IEEE Commun. Magazine, vol. 45, no 8, pp.87-93, Aug. 2007.

2. S. Sesia, G. Caire, and G. Vivier, "Lossy transmission over slow-fading AWGN channels: A comparison of progressive, superposition and hybrid approaches," in Proc. IEEE Int. Symp. Info. Theory, Adelaide, Australia, pp. 224-228, Sep. 2005.

3. F. Etemadi and H. Jafarkhani, "Optimal layered transmission over quasi-static fading channels," in Proc. IEEE Int. Symp. Info. Theory, Seattle, WA, Jul. 2006, pp. 1051-1055. 
4. C. Ng, D. Gndz, A. Goldsmith, and E. Erkip, "Recursive power allocation in Gaussian layered broadcast coding with successive refinement," in Proc. IEEE Int. Conf. Commun. (ICC), Glasgow, Scotland, U.K., Jun. 2007, pp. 889-896.

5. C. Ng, D. Gndz, A. Goldsmith, and E. Erkip, "Minimum expected distortion in Gaussian layered broadcast coding with successive refinement," in Proc. IEEE Int. Symp. Info. Theory, Nice, France, Jun. 2007, pp. 2226-2230.

6. J. She, X. Yu, Pin-Han Ho, and E. -H. Yang, "A Cross-Layer Design Framework for Robust IPTV Services over IEEE 802.16 Networks", IEEE Journal of Selected Areas on Communications (JSAC), vol. 27, No. 2, Feb. 2009, pp. 235 - 245.

7. Y. S. Chan, J. W. Modestino,Q. Qu and X. Fan, "An End-to-End Embedded Approach for Multicast/Broadcast of Scalable Video over Multiuser CDMA Wireless Networks", IEEE Trans. Multimedia, vol. 9, no. 3, pp. 655-667, Apr. 2007.

8. Z. Cakareski, N. Ahmed,A.Dhar and B. Aazhang,"Multilevel coding of broadcast video over wireless channels", in Proc. of IEEE Int. Conf. on Acoustics, Speech, and Signal Processing, (ICASSP '02), vol.3, pp. I-2797- III-2800, Aug. 2002.

9. C. Tian, A. Steiner, S. Shamai (Shitz) and S. N. Diggavi, "Successive Refinement Via Broadcast: Optimizing Expected Distortion of a Gaussian Source Over a Gaussian Fading Channel", IEEE Trans. Info. Theory, vol. 54, no. 7, pp.2903-2918, Jul. 2008.

10. M. R. Chari, F. Ling, A. Mantravadi, R. Krishnamoorthi, R. Vijayan, G. K. Walker and Rob Chandhok, "FLO Physical Layer: An Overview", IEEE Trans. Broadcasting, vol. 53, no. 1, pp. 145-160, Mar. 2007

11. Qualcomm Incorporated, "Hierarchical Coding With Multiple Antennas In A Wireless Communication System", WO/2005/032035, Patent Application, Patent Cooperation Treaty, Sep. 2004.

12. T. M. Cover, "Broadcast channels," IEEE Trans. Info. Theory, vol. 18, no. 1, pp. 2-14, Jan. 1972.

13. S. Shamai (Shitz), "A broadcast strategy for the Gaussian slowly fading channel," in Proc. IEEE Int. Symp. Info. Theory, June 1997, p. 150.

14. S. Shamai (Shitz) and A. Steiner, "A broadcast approach for a single-user slowly fading MIMO channel," IEEE Trans. Info. Theory, vol. 49, no. 10, pp. 2617-2635, Oct. 2003. 